\title{
MOMENT AND MGF CONVERGENCE OF OVERSHOOTS AND UNDERSHOOTS FOR LÉVY INSURANCE RISK PROCESSES
}

\author{
HYUN SUK PARK, * Australian National University and \\ Pohang University of Science and Technology \\ ROSS MALLER, ${ }^{* *}$ Australian National University
}

\begin{abstract}
This paper is concerned with the finiteness and large-time behaviour of moments of the overshoot and undershoot of a high level, and of their moment generating functions (MGFs), for a Lévy process which drifts to $-\infty$ almost surely. This provides information relevant to quantities associated with the ruin of an insurance risk process. Results of Klüppelberg, Kyprianou, and Maller (2004) and Doney and Kyprianou (2006) for asymptotic overshoot and undershoot distributions in the class of Lévy processes with convolution equivalent canonical measures are shown to have moment and MGF convergence extensions.
\end{abstract}

Keywords: Insurance risk process; overshoot; undershoot; Lévy process; ladder height process; subexponential and convolution equivalent distributions

2000 Mathematics Subject Classification: Primary 60G51; 60K05

Secondary 91B30; 60F05

\section{Introduction}

Suppose that $X=\left\{X_{t}: t \geq 0\right\}, X_{0}=0$, is a Lévy process defined on $(\Omega, \mathcal{F}, \mathrm{P})$ with triplet $\left(\gamma, \sigma^{2}, \Pi_{X}\right), \Pi_{X}$ being the Lévy measure of $X$. Thus, the characteristic function of $X$ is given by the Lévy-Khintchine representation, $\mathrm{E}\left[\exp \left\{\mathrm{i} \theta X_{t}\right\}\right]=\mathrm{e}^{t \Psi(\theta)}$, where

$$
\Psi(\theta)=\mathrm{i} \theta \gamma-\frac{\sigma^{2} \theta^{2}}{2}+\int_{\mathbb{R} \backslash\{0\}}\left(\mathrm{e}^{\mathrm{i} \theta x}-1-\mathrm{i} \theta x \mathbf{1}_{\{|x|<1\}}\right) \Pi_{X}(\mathrm{~d} x) \quad \text { for } \theta \in \mathbb{R}, t \geq 0 .
$$

We will consider limiting distributions and limits of moments of the overshoot and undershoot of the process above a high level, and the undershoot from the previous maximum, viz. the quantities $X_{\tau(u)}-u, u-X_{\tau(u)-}$, and $u-\bar{X}_{\tau(u)-}$, conditional on $\tau(u)<\infty$, where $\bar{X}_{t}=\sup _{0<s \leq t} X_{s}$ and $\tau(u)$ is the first passage time above level $u$ :

$$
\tau(u)=\inf \left\{t \geq 0: X_{t}>u\right\}, \quad u>0 .
$$

Throughout, we assume that the process drifts to $-\infty$ almost surely (a.s.). This corresponds to an insurance risk model with premiums and other income producing a downward drift in $X$, while claims are represented by positive jumps. Thus, 'ruin' occurs at a positive level $u$ if

Received 3 October 2007; revision received 7 July 2008.

* Postal address: Pohang Mathematics Institute, POSTECH, Pohang 790-784, South Korea.

Email address: hspark@postech.ac.kr

** Postal address: School of Finance and Applied Statistics and Centre for Mathematics and its Applications, Australian National University, Canberra 0200, Australia. Email address: ross.maller@anu.edu.au 
this level is reached by $X$. We wish to analyse the asymptotic behaviour of distributions and moments of the overshoot and undershoot, conditional on first passage occurring in a finite time, as the reserve level $u$ is increased to $\infty$.

In this analysis, throughout, we will follow the notation of Klüppelberg et al. [9] for the most part. Thus, $\left(H_{t}\right)_{t \geq 0}$ will define the ascending ladder height subordinator of $X$, which is defective under the assumption that $\lim _{t \rightarrow \infty} X_{t}=-\infty$ a.s. (which we will always assume in this paper), taking the value $+\infty$ once $X$ exceeds its all-time maximum. The (proper) descending ladder height subordinator is denoted by $\left(\hat{H}_{t}\right)_{t \geq 0}$ (taking positive values; we depart from the notation of [9] here, in favour of the setup in Doney and Kyprianou [3]).

Continuing, we define the following renewal measure of the (defective, when $\lim _{t \rightarrow \infty} X_{t}=$ $-\infty$ a.s.) process $H$ :

$$
V(\mathrm{~d} x)=\int_{(0, \infty)} \mathrm{P}\left(H_{t} \in \mathrm{d} x\right) \mathrm{d} t=\int_{(0, \infty)} \mathrm{P}\left(\mathscr{H}_{t} \in \mathrm{d} x\right) \mathrm{e}^{-q t} \mathrm{~d} t \quad \text { for } x \geq 0,
$$

where $\mathscr{H}$ is a nondefective process and $H$ is obtained from $\mathscr{H}$ by exponential killing with rate $q>0$. Here $V(x)$ is finite for each $x \geq 0$, as is the renewal measure $\hat{V}$ of the downward ladder height process $\hat{H}$, satisfying

$$
\hat{V}(\mathrm{~d} x)=\int_{(0, \infty)} \mathrm{P}\left(\hat{H}_{t} \in \mathrm{d} x\right) \mathrm{d} t \quad \text { for } x \geq 0 .
$$

We denote the Lévy measure of $\mathscr{H}$ by $\Pi_{\mathscr{H}}$, and similarly for $\hat{H}$. The tails of $\Pi_{X}$ are

$$
\bar{\Pi}_{X}^{+}(x)=\Pi_{X}\{(x, \infty)\}, \quad \bar{\Pi}_{X}^{-}(x)=\Pi_{X}\{(-\infty,-x)\},
$$

and

$$
\bar{\Pi}_{X}(x)=\bar{\Pi}_{X}^{+}(x)+\bar{\Pi}_{X}^{-}(x), \quad x>0,
$$

with similar $\bar{\Pi}_{\mathcal{H}}(x)$ and $\bar{\Pi}_{\hat{H}}(x)$ notation for $\mathscr{H}$ and $\hat{H}$. From [9, Corollary 2.9] we have the following convolution identity:

$$
\mathrm{P}\left(X_{\tau(u)}-u>x, \tau(u)<\infty\right)=\int_{(0, u]} \bar{\Pi}_{\mathscr{H}}(x+u-y) \mathrm{d} V(y), \quad x>0, u>0 .
$$

A natural case to investigate is when the positive tail of the Lévy measure of $X$ (or of the ascending ladder height measure) is subexponential (or, more generally, convolution equivalent in the sense defined below). We only briefly introduce the main ideas here; background, rationale for these assumptions, and examples are given in [9]. We say that a distribution $F$ on $[0, \infty)$ with tail $\bar{F}$ belongs to the class $\mathcal{L}^{(\alpha)}, \alpha \geq 0$, if

$$
\lim _{u \rightarrow \infty} \frac{\bar{F}(u-x)}{\bar{F}(u)}=\mathrm{e}^{\alpha x} \text { for } x \in(-\infty, \infty) .
$$

Tail functions $\bar{F}$ such that $\bar{F}(\log u)$ is regularly varying with index $-\alpha, \alpha \geq 0$, as $u \rightarrow \infty$, are in $\mathcal{L}^{(\alpha)}$. With ' $*$ ' denoting the convolution, a distribution $F$ is said to be convolution equivalent, i.e. in the class $\delta^{(\alpha)}, \alpha \geq 0$, if $F \in \mathcal{L}^{(\alpha)}$ and, in addition,

$$
\lim _{x \rightarrow \infty} \frac{\overline{F^{2 *}}(x)}{\bar{F}(x)}=2 \delta_{\alpha}(F)<\infty,
$$


where $\delta_{\alpha}(F):=\int_{(0, \infty)} \mathrm{e}^{\alpha x} F(\mathrm{~d} x)$. The parameter $\alpha$ is referred to as the index of the class. When $\alpha=0, \delta:=\delta^{(0)}$ is the class of subexponential distributions. The subexponential or, more generally, the convolution equivalent distributions are used in modelling heavy-tailed data, such as occur in insurance applications; we refer the reader to [5], [6], [10], and the references therein for discussion and properties. Distributions in the class $\delta^{(\alpha)}$ for $\alpha \geq 0$ are "near to exponential' in the sense that their tails are only slightly modified exponential. An important class of distributions which are convolution equivalent or subexponential for some values of the parameters is the generalised inverse Gaussian distributions; see [8].

We can take the tail of any Lévy canonical measure, assumed nonzero on some interval contained in $(0, \infty)$, to be the tail of a distribution function on $[0, \infty)$, after renormalisation. With this convention, we then say that the measure is in $\mathcal{L}^{(\alpha)}$ or $f^{(\alpha)}$ if this is true for the distribution with the corresponding (renormalised) tail. As in Klüppelberg et al. [9], we will restrict ourselves to the nonlattice case, with the understanding that the alternative can be handled by obvious modifications.

In what follows, we set out results for the overshoot in Section 2 and for the undershoots in Section 3. Each section gives conditions for finiteness of moments and of the MGF of the respective quantity, as well as asymptotic results for these as the level $u$ tends to $\infty$. All proofs are collected in Section 4.

\section{Finiteness and limit behaviour of moments of the overshoot}

Throughout, we will assume that $\lim _{t \rightarrow \infty} X_{t}=-\infty$ a.s. Doney and Maller [4] showed that this is the case if and only if $\mathrm{E}\left[X_{1}\right]<0$ when $\mathrm{E}\left[\left|X_{1}\right|\right]<\infty$, or, when $\mathrm{E}\left[\left|X_{1}\right|\right]=\infty$,

$$
\int_{(1, \infty)} \frac{y}{A_{-}(y)} \Pi_{X}(\mathrm{~d} y)<\infty
$$

where

$$
A_{-}(x):=1+\int_{1}^{x} \bar{\Pi}_{X}^{-}(y) \mathrm{d} y, \quad x \geq 1 .
$$

Our first result shows how this is modified to obtain finiteness of moments of the overshoot. Note that we allow the possibility of $\mathrm{E}\left[X_{1}^{-}\right]=\infty$ in most of our results. Assuming that $\mathrm{E}\left[X_{1}^{-}\right]<\infty$ would undesirably limit the growth of the 'income' side of $X$ in the risk process. However, we do need (2.1). In Theorem 2.1, below, part (a) is proved in [4] (with a sign change).

Theorem 2.1. Assume that $\lim _{t \rightarrow \infty} X_{t}=-\infty$ a.s.

(a) Take $\beta>0$. Then we have, for each $u>0$,

$$
\mathrm{E}\left[\left(X_{\tau(u)}-u\right)^{\beta} \mid \tau(u)<\infty\right]<\infty \text { if and only if } \int_{(1, \infty)} \frac{x^{\beta+1}}{A_{-}(x)} \Pi_{X}(\mathrm{~d} x)<\infty .
$$

(b) Take $\theta>0$. Then we have, for each $u>0$,

$$
\mathrm{E}\left[\exp \left\{\theta\left(X_{\tau(u)}-u\right)\right\} \mid \tau(u)<\infty\right]<\infty \text { if and only if } \int_{(1, \infty)} \mathrm{e}^{\theta x} \Pi_{X}(\mathrm{~d} x)<\infty .
$$

Remarks. (i) Equivalences for convergence of integrals such as (2.2) and (2.3) in terms of the marginal distribution of $X_{1}$ can be written directly from [11, p. 159]. 
(ii) Of course, since $u$ is fixed in Theorem 2.1, the convergence of the integrals in (2.2) and (2.3) are also necessary and sufficient for finiteness of

$$
\mathrm{E}\left[X_{\tau(u)}^{\beta} \mid \tau(u)<\infty\right] \text { and } \mathrm{E}\left[\exp \left\{\theta X_{\tau(u)}\right\} \mid \tau(u)<\infty\right] .
$$

But it is convenient to work in terms of the overshoot; cf. Theorem 2.2, below.

(iii) Convergence of the integral in (2.2) is equivalent to that of $\int_{1}^{\infty}\left(x^{\beta} \bar{\Pi}^{+}(x) / A_{-}(x)\right) \mathrm{d} x$, when $\beta>0$, as can be shown by integrating by parts. This form clearly shows that the convergences in (2.2) occur when the negative tail of $\Pi$ dominates the positive tail appropriately, and may be slightly more convenient in applications.

(iv) It is surprising that no denominator $A_{-}(x)$ is needed in (2.3), as might be expected in view of (2.1) and (2.2). When $\mathrm{E}\left[\left|X_{1}\right|\right]<\infty$, then $A_{-}(\infty)<\infty$, and $A_{-}(x)$ can simply be omitted in (2.2) also, leaving an integral equivalent to $\mathrm{E}\left[\left(X_{1}^{+}\right)^{\beta+1}\right]$. Convergence of the integral in (2.3) is of course equivalent to $\mathrm{E}\left[\exp \left\{\theta X_{1}^{+}\right\}\right]<\infty$.

For our next result, we are interested in the behaviour of the moments and MGF of the overshoot as $u \rightarrow \infty$. At this stage, we bring in the subexponential/convolution equivalent assumptions. For $\alpha \geq 0$, we assume the same conditions as in [9], namely, that

$$
\begin{gathered}
\lim _{t \rightarrow \infty} X_{t}=-\infty \quad \text { a.s., } \quad \bar{\Pi}_{X}^{+}(0)>0, \\
\bar{\Pi}_{\mathscr{H}} \in \mathcal{f}^{(\alpha)}, \quad \text { and } \quad \mathrm{e}^{-q} \delta_{\alpha}(\mathscr{H})<1 .
\end{gathered}
$$

(Recall that $q>0$ is defined in (1.1).) The case in which $\Pi_{X}$ is concentrated on $(-\infty, 0)$, i.e. $X$ is spectrally negative, is rather easy to handle, so we exclude it, while the assumption that $\bar{\Pi}_{\mathscr{H}} \in f^{(\alpha)}$ means that $\delta_{\alpha}(\mathscr{H}):=\mathrm{E}\left[\exp \left\{\alpha \mathscr{H}_{1}\right\}\right]$ is finite; cf. (1.4). Keeping $\mathrm{e}^{-q} \delta_{\alpha}(\mathscr{H})<1$ means that we are in the non-Cramér case. The asymptotic behaviour of $\tau(u)$ and associated quantities in the Cramér case is quite different; see [9, Remarks 4.6 and 5.2] and also [7].

Under (2.4), we have, from [9, Proposition 2.5 and Lemma 3.5], for $\alpha \geq 0$,

$$
\lim _{u \rightarrow \infty} \frac{\bar{V}(u)}{\bar{\Pi}_{\mathscr{H}}(u)}=\delta_{\alpha}^{2}(V) \text { and } \lim _{u \rightarrow \infty} \frac{\mathrm{P}(\tau(u)<\infty)}{\bar{\Pi}_{\mathcal{H}}(u)}=q \delta_{\alpha}^{2}(V),
$$

where $\bar{V}(u):=V(\infty)-V(u)=1 / q-V(u)$ and $\delta_{\alpha}(V)=\int_{(0, \infty)} \mathrm{e}^{\alpha x} V(\mathrm{~d} x)$, while, for $\alpha>0$ (see [9, Proposition 5.3]),

$$
\lim _{u \rightarrow \infty} \frac{\bar{\Pi}_{X}^{+}(u)}{\bar{\Pi}_{\mathscr{H}}(u)}=\hat{\kappa}(-\mathrm{i} \alpha),
$$

where $\hat{\kappa}(\theta):=\log \mathrm{E}\left[\exp \left\{\mathrm{i} \theta \hat{H}_{1}\right\}\right]$ for $\theta \in \mathbb{R}$. The following identity, due to Vigon [12], will also be very useful:

$$
\bar{\Pi}_{\mathscr{H}}(u)=\int_{(0, \infty)} \bar{\Pi}_{X}^{+}(u+y) \hat{V}(\mathrm{~d} y), \quad u>0 .
$$

When $\bar{\Pi}_{\mathscr{H}} \in \mathcal{f}^{(\alpha)}$ for an $\alpha>0$, we can define a distribution function $G$ on $[0, \infty)$ in terms of its tail by

$$
\bar{G}(x)=\frac{\mathrm{e}^{-\alpha x}}{q}\left(q-\log \delta_{\alpha}(\mathcal{H})+\int_{(x, \infty)}\left(\mathrm{e}^{\alpha y}-\mathrm{e}^{\alpha x}\right) \Pi_{\mathscr{H}}(\mathrm{d} y)\right), \quad x \geq 0 .
$$


The function $G$ is then a proper cumulative distribution function (total mass 1). When $\alpha=0$, we take $\bar{G}(x)=0$, i.e. $G$ is improper with all its mass at $+\infty$. From Klüppelberg et al. [9, Theorem 4.2], the $G$ thus defined is the limiting distribution of $X_{\tau(u)}-u$, conditional on $\tau(u)<\infty$, as $u \rightarrow \infty$, when (2.4) obtains and $\alpha \geq 0$.

Given all this setup, we can prove the following theorem.

Theorem 2.2. Assume that (2.4) holds for an $\alpha>0$.

(a) Fix $\beta>0$. Then we have

$$
\lim _{u \rightarrow \infty} \mathrm{E}\left[\left(X_{\tau(u)}-u\right)^{\beta} \mid \tau(u)<\infty\right]=\int_{(0, \infty)} x^{\beta} G(\mathrm{~d} x) .
$$

(b) Fix $0<\theta<\alpha$. Then we have

$$
\lim _{u \rightarrow \infty} \mathrm{E}\left[\exp \left\{\theta\left(X_{\tau(u)}-u\right)\right\} \mid \tau(u)<\infty\right]=\int_{(0, \infty)} \mathrm{e}^{\theta x} G(\mathrm{~d} x) .
$$

Remarks. (i) When (2.4) holds and $\alpha=0$, the limits in (2.9) and (2.10) are $+\infty$ for any $\beta>0$ and $\theta>0$. This is easily proved by Fatou's lemma, since $\lim _{u \rightarrow \infty} \mathrm{P}\left(X_{\tau(u)}-u>x \mid \tau(u)<\right.$ $\infty)=1$ for all $x>0$ in the case in which $\alpha=0$. We will not consider this case further in the present paper.

(ii) Note that the integrals in (2.9) and (2.10) are finite, under (2.4), when $\alpha>0$. In fact, it is easy to check from (2.8) that $\bar{G}(x)=O\left(\mathrm{e}^{-\alpha x}\right)$ as $x \rightarrow \infty$.

(iii) Given that the limiting distribution, as derived in [9, Theorem 4.2], is $G$, as in (2.8), establishing (2.9) and (2.10) is essentially a matter of taking a limit under an integral. This is not a trivial matter in the present context, because, although uniform bounds on certain quantities are available from regularly varying theory (cf. (4.5) and (4.6), below), straightforward application of the dominated convergence theorem is not possible. The uniform bound contains an arbitrary $\varepsilon>0$ in the exponent, and a distribution function $F$ in the class $f^{(\alpha)}$ is heavy tailed in the sense that the integral $\int_{(0, \infty)} \mathrm{e}^{(\alpha+\varepsilon) x} F(\mathrm{~d} x)$ is infinite for any $\varepsilon>0$ (see [9]). The claimed moment convergences have to be shown by careful analysis relying strongly on the special properties of convolution equivalent distributions.

(iv) The results in Theorem 2.2 are very general. Drift of $X$ to $-\infty$ does not require a finite mean for $X$, only (2.1). Assumption (2.4) allows for a wide class of 'claim' processes, represented by the positive tail of $X$, but (2.4) for $\alpha>0$ requires integrability of $\Pi_{X}^{+}(\mathrm{d} x)$ against $\mathrm{e}^{\alpha x}$. Note that we assume no more than (2.4), even for the moment convergences.

The rather abstract quantities, $\delta_{\alpha}(\mathscr{H}), \Pi_{\mathscr{H}}(y)$, etc., appearing in (2.8) and elsewhere can be given quite concrete values in special cases, especially in the case in which $X$ is spectrally positive. A number of applications examples, which generalise many results in the previous literature, are given in [3] and [9]. Using our results, expressions for limiting moments of the overshoot and undershoot are easily read off from these, so we will not go into the details here.

\section{Convergence of distributions and moments of the undershoot}

Now we consider quantities related to the undershoots. A very recent 'quintuple law' of Doney and Kyprianou [3, Theorem 3] gives the following result, when translated to our notation: 


$$
\begin{aligned}
& \text { for } \gamma>0, v>0,0<y \leq u \wedge v, g \geq 0, \text { and } h \geq 0, \\
& \begin{array}{l}
\mathrm{P}\left(\tau(u)-\bar{G}_{\tau(u)-} \in \mathrm{d} g, \bar{G}_{\tau(u)-} \in \mathrm{d} h, X_{\tau(u)}-u \in \mathrm{d} \gamma, u-X_{\tau(u)-} \in \mathrm{d} v, u-\bar{X}_{\tau(u)-} \in \mathrm{d} y\right) \\
\quad=|V(u-\mathrm{d} y, \mathrm{~d} h)| \hat{V}(\mathrm{~d} v-y, \mathrm{~d} g) \Pi_{X}(\mathrm{~d} \gamma+v),
\end{array}
\end{aligned}
$$

where, recall, $\bar{X}_{t}=\sup _{0<s \leq t} X_{s}$ and $\bar{G}_{t}=\sup \left\{s \leq t: X_{s}=\bar{X}_{s}\right\}$. Here $V(\cdot, \cdot)$ and $\hat{V}(\cdot, \cdot)$ are the bivariate renewal functions of the ascending and descending ladder height and inverse local time subordinators. Equation (3.1) plays a crucial role in our calculations. Integrating out the variables gives

$$
\begin{aligned}
\mathrm{P}(u & \left.-X_{\tau(u)-} \in \mathrm{d} v, \tau(u)<\infty\right) \\
& =-\int_{g \geq 0} \int_{h \geq 0} \int_{\gamma>0} \int_{y \in(0, u \wedge v]} V(u-\mathrm{d} y, \mathrm{~d} h) \hat{V}(\mathrm{~d} v-y, \mathrm{~d} g) \Pi_{X}(\mathrm{~d} \gamma+v) \\
& =-\bar{\Pi}_{X}^{+}(v) \int_{y \in(0, u \wedge v]} \mathrm{d}_{y} V(u-y) \mathrm{d}_{v} \hat{V}(v-y), \quad u>0, v>0,
\end{aligned}
$$

where $\mathrm{d}_{y} V(x-y):=V(x-\mathrm{d} y)$ and $\mathrm{d}_{v} \hat{V}(v-y):=\hat{V}(\mathrm{~d} v-y)$, with $V$ and $\hat{V}$ as defined in (1.1) and (1.2). We can use (3.2) to check finiteness and convergence of moments of the undershoot.

Theorem 3.1. Assume that $\lim _{t \rightarrow \infty} X_{t}=-\infty$ a.s.

(a) Fix $\beta>0$ and $u>0$. Then we have

$$
\mathrm{E}\left[\left(u-X_{\tau(u)-}\right)^{\beta} \mid \tau(u)<\infty\right]<\infty \text { if } \int_{1}^{\infty} \frac{x^{\beta+1}}{A_{-}(x)} \Pi_{X}(\mathrm{~d} x)<\infty .
$$

The converse is true if, in addition, we assume that $\mathrm{E}\left[\left|X_{1}\right|\right]<\infty$.

(b) Fix $\theta>0$ and $u>0$. Then we have

$$
\mathrm{E}\left[\exp \left\{\theta\left(u-X_{\tau(u)-}\right)\right\} \mid \tau(u)<\infty\right]<\infty \text { if } \int_{1}^{\infty} \frac{x \mathrm{e}^{\theta x}}{A_{-}(x)} \Pi_{X}(\mathrm{~d} x)<\infty .
$$

Remarks. Finding a general necessary and sufficient condition for the finiteness of the expectations in (3.3) and (3.4), instead of the partial solution given in Theorem 3.1, seems rather elusive, depending on some fine structure properties of the renewal function of the negative ladder height process, $\hat{V}(x)$. Note that in (3.4) we have to include a factor of $x / A_{-}(x)$ in the integral, whereas this was not needed in (2.3).

The next theorem gives the limiting (improper) distribution of the undershoot and limits of its pseudomoments as $u \rightarrow \infty$.

Theorem 3.2. Assume that (2.4) holds for an $\alpha>0$. Then,

(a) for all $x>0$, we have

$$
\begin{aligned}
\lim _{u \rightarrow \infty} \mathrm{P} & \left(u-X_{\tau(u)-}>x \mid \tau(u)<\infty\right) \\
= & \frac{1}{q \delta_{\alpha}(V)}+\frac{1}{q} \int_{(x, \infty)}\left(\mathrm{e}^{\alpha y}-1\right) \Pi_{\mathcal{H}}(\mathrm{d} y) \\
& -\frac{1}{q} \int_{y \in(0, x)} \int_{v \in(x-y, \infty)} \bar{\Pi}_{X}^{+}(v+y) \hat{V}(\mathrm{~d} v) \mathrm{d}\left(\mathrm{e}^{\alpha y}\right)
\end{aligned}
$$


(b) for $\beta>0$, we have

$$
\lim _{u \rightarrow \infty} u^{-\beta} \mathrm{E}\left[\left(u-X_{\tau(u)-}\right)^{\beta} \mid \tau(u)<\infty\right]=\frac{1}{q \delta_{\alpha}(V)} ;
$$

(c) for $0<\theta<\alpha$, we have

$$
\lim _{u \rightarrow \infty} \mathrm{e}^{-\theta u} \mathrm{E}\left[\exp \left\{\theta\left(u-X_{\tau(u)-}\right)\right\} \mid \tau(u)<\infty\right]=\frac{\hat{\kappa}(-\mathrm{i} \alpha) \delta_{\alpha-\theta}(V)}{q \hat{\kappa}(-\mathrm{i} \alpha+\mathrm{i} \theta) \delta_{\alpha}^{2}(V)} .
$$

Remark. The limiting function on the right-hand side of (3.5) is an improper distribution with mass $1 / q \delta_{\alpha}(V)$ at $\infty$. In a way this captures the intuition already expounded in [3] that ruin may occur by a very large jump at a finite time or by an accumulation of smaller jumps over a longer period of time.

While Doney and Kyprianou [3] gave in their Theorems 10 and 11 expressions for the limit of the joint distribution of the overshoot and undershoot in the sense of vague convergence, this does not give the limiting distribution (or the limiting improper distribution in the case of the undershoot) because there is still the problem of taking the limit through the integrals in their expressions. As shown in our proofs, this is a delicate exercise, and the more so in the case of convergence of moments or MGFs.

Compared with Theorem 2.2, we see quite a different behaviour for the pseudomoments of the undershoots; they grow with level $u$ at an algebraic or exponential rate, as shown in (3.6) and (3.7). A similar phenomenon occurs for the undershoot from the previous maximum, as we show next. Integration of (3.1) and use of (2.7) gives its distribution in the form

$$
\mathrm{P}\left(u-\bar{X}_{\tau(u)-} \in \mathrm{d} y, \tau(u)<\infty\right)=\bar{\Pi}_{\mathcal{H}}(y) \mathrm{d}_{y} \bar{V}(u-y), \quad 0 \leq y \leq u .
$$

Of course, this undershoot lies between 0 and $u$, so it has finite moments of all orders for finite $u$. We examine the limiting behaviour of its distribution and moments in the next theorem.

Theorem 3.3. Assume that (2.4) holds for an $\alpha>0$. Then,

(a) for $x>0$, we have

$$
\lim _{u \rightarrow \infty} \mathrm{P}\left(u-\bar{X}_{\tau(u)-} \leq x \mid \tau(u)<\infty\right)=\frac{\alpha}{q} \int_{0}^{x} \mathrm{e}^{\alpha y} \bar{\Pi}_{\mathcal{H}}(y) \mathrm{d} y ;
$$

(b) for $\beta>0$, we have

$$
\lim _{u \rightarrow \infty} u^{-\beta} \mathrm{E}\left[\left(u-\bar{X}_{\tau(u)-}\right)^{\beta} \mid \tau(u)<\infty\right]=\frac{1}{q \delta_{\alpha}(V)} ;
$$

(c) for $0<\theta<\alpha$, we have

$$
\lim _{u \rightarrow \infty} \mathrm{e}^{-\theta u} \mathrm{E}\left[\exp \left\{\theta\left(u-\bar{X}_{\tau(u)-}\right)\right\} \mid \tau(u)<\infty\right]=\frac{\delta_{\alpha-\theta}(V)}{q \delta_{\alpha}^{2}(V)} .
$$

Remark. The distribution on the right-hand side of (3.9) has mass $1-\delta_{\alpha}(\mathcal{H}) / q=1 / q \delta_{\alpha}(V)$ at $\infty$, like that in (3.5), though they are quite different distributions. Equations (3.6) and (3.10) give the same limit for the $\beta$-moments of the undershoot; but, (3.7), which reflects the influence of $\hat{V}$ in (3.2), is quite different to (3.11). Generally speaking, the undershoots have heaviertailed limiting distributions than the overshoot, reflecting the possibility of large jumps across the boundary as a result of the heavy-tailed convolution-equivalent assumption. 


\section{Proofs}

Proof of Theorem 2.1. Part (a) follows from Theorem 5 of [4] after making a sign reversal, i.e. replacing $X$ by $-X$. (Note that the $A(x)$ used in [4] can be replaced by an integral concerning the positive tail of $\Pi$ (see [4, Equation (1.19)]), which becomes $A_{-}(x)$ in our present notation, after a sign reversal.)

To prove part (b), use (1.3) to obtain, for $u>0$ and $\theta>0$,

$$
\int_{1}^{\infty} \mathrm{e}^{\theta x} \mathrm{P}\left(X_{\tau(u)}-u>x, \tau(u)<\infty\right) \mathrm{d} x=\int_{y \in(0, u]} \int_{u-y+1}^{\infty} \mathrm{e}^{\theta(x-u+y)} \bar{\Pi}_{\mathcal{H}}(x) \mathrm{d} x V(\mathrm{~d} y) .
$$

Let $\hat{A}(x):=\int_{0}^{x} \bar{\Pi}_{\hat{H}}(y) \mathrm{d} y$. From [9, Equation 7.21], there are constants $c_{ \pm} \in(0, \infty)$ such that

$$
c_{-} \int_{(x, \infty)} \frac{z-x}{\hat{A}(z-x)} \Pi_{X}(\mathrm{~d} z) \leq \bar{\Pi}_{\mathcal{H}}(x) \leq c_{+} \int_{(x, \infty)} \frac{z-x}{\hat{A}(z-x)} \Pi_{X}(\mathrm{~d} z),
$$

whenever $x$ is greater than or equal to some $\eta>0$. To use this in (4.1), consider

$$
\begin{aligned}
\int_{u-y+1}^{\infty} & \mathrm{e}^{\theta(x-u+y)} \int_{z \in(x, \infty)} \frac{z-x}{\hat{A}(z-x)} \Pi_{X}(\mathrm{~d} z) \mathrm{d} x \\
& =\int_{z \in(u-y+1, \infty)} \int_{u-y+1}^{z} \mathrm{e}^{\theta(x-u+y)} \frac{z-x}{\hat{A}(z-x)} \mathrm{d} x \Pi_{X}(\mathrm{~d} z) \\
& =\int_{(u-y+1, \infty)} \mathrm{e}^{\theta(z-u+y)} \int_{0}^{z-u+y-1} \mathrm{e}^{-\theta x} \frac{x}{\hat{A}(x)} \mathrm{d} x \Pi_{X}(\mathrm{~d} z) .
\end{aligned}
$$

Here we give $x / \hat{A}(x)$ its limiting value at 0 , at 0 , namely,

$$
\left(\lim _{x \downarrow 0} x^{-1} \int_{0}^{x} \bar{\Pi}_{\hat{H}}(y) \mathrm{d} y\right)^{-1}=\left(\bar{\Pi}_{\hat{H}}(0+)\right)^{-1},
$$

which is in $[0, \infty)$ since $X$ drifts to $-\infty$ a.s. and $\hat{H}$ is not trivial. The inner integral in (4.2) is bounded above by

$$
c_{\theta}:=\int_{0}^{\infty} \mathrm{e}^{-\theta x} \frac{x}{\hat{A}(x)} \mathrm{d} x
$$

and this is finite because $\hat{A}(\infty)=\int_{0}^{\infty} \bar{\Pi}_{\hat{H}}(y) \mathrm{d} y>0$. Thus, the right-hand side of (4.1) is bounded above by

$$
\begin{aligned}
c_{+} c_{\theta} & \int_{(0, u]} \mathrm{e}^{-\theta(u-y)} \int_{z \in(u-y+1, \infty)} \mathrm{e}^{\theta z} \Pi_{X}(\mathrm{~d} z) V(\mathrm{~d} y) \\
& \leq c_{+} c_{\theta} \int_{(0, u]} \mathrm{e}^{-\theta(u-y)} V(\mathrm{~d} y) \int_{z \in(1, \infty)} \mathrm{e}^{\theta z} \Pi_{X}(\mathrm{~d} z) .
\end{aligned}
$$

The right-hand side of this is finite when the integral in (2.3) is finite, so the expectation in (2.3) is finite. 
Conversely, suppose that the expectation in (2.3) is finite. Using (4.1) and (4.2), we obtain

$$
\begin{aligned}
\int_{1}^{\infty} & \mathrm{e}^{\theta x} \mathrm{P}\left(X_{\tau(u)}-u>x, \tau(u)<\infty\right) \mathrm{d} x \\
& \geq c_{-} \int_{(0, u / 2]} \mathrm{e}^{-\theta(u-y)} \int_{(2(u-y+1), \infty)} \mathrm{e}^{\theta z} \Pi_{X}(\mathrm{~d} z) \int_{0}^{u-y+1} \mathrm{e}^{-\theta x} \frac{x}{\hat{A}(x)} \mathrm{d} x V(\mathrm{~d} y) \\
& \geq c_{-} \mathrm{e}^{-\theta u} \int_{(2(u+1), \infty)} \mathrm{e}^{\theta z} \Pi_{X}(\mathrm{~d} z) \int_{(0, u / 2]} \mathrm{e}^{\theta y} \int_{0}^{u / 2} \mathrm{e}^{-\theta x} \frac{x}{\hat{A}(x)} \mathrm{d} x V(\mathrm{~d} y),
\end{aligned}
$$

from which we see that the integral in (2.3) is finite.

Proof of Theorem 2.2. Assume that (2.4) holds for an $\alpha>0$. It suffices to prove part (b), as part (a) then follows by standard methods.

Take $\theta \in(0, \alpha), u>0$, and $y \in(0, u]$. Define

$$
I(u, y):=\int_{1}^{\infty} \bar{\Pi}_{\mathscr{H}}\left(\theta^{-1} \log x+u-y\right) \mathrm{d} x .
$$

Then by (1.3) we have

$$
\begin{aligned}
\frac{1}{\bar{\Pi}_{\mathscr{H}}(u)} & \mathrm{E}\left[\exp \left\{\theta\left(X_{\tau(u)}-u\right)\right\}-1 ; \tau(u)<\infty\right] \\
= & \frac{1}{\bar{\Pi}_{\mathscr{H}}(u)} \int_{1}^{\infty} \int_{(0, u]} \bar{\Pi}_{\mathscr{H}}\left(\theta^{-1} \log x+u-y\right) \mathrm{d} x V(\mathrm{~d} y) \\
= & \frac{1}{\bar{\Pi}_{\mathscr{H}}(u)} \int_{(0, u]} I(u, y) V(\mathrm{~d} y) \\
= & \int_{(0, u]}\left(\frac{I(u, y)}{\bar{\Pi}_{\mathscr{H}}(u)}-I(y)\right) V(\mathrm{~d} y)+\int_{(0, u]} I(y) V(\mathrm{~d} y),
\end{aligned}
$$

where

$$
I(y):=\mathrm{e}^{\alpha y} \int_{1}^{\infty} x^{-\alpha / \theta} \mathrm{d} x=\frac{\theta \mathrm{e}^{\alpha y}}{\alpha-\theta} .
$$

We need some bounds for ratios like the one in (4.4). Let $\bar{F}(x):=\bar{\Pi}_{\mathscr{H}}(\log x)$, which is well defined and positive for $x>1$. The class $f^{(\alpha)}$ is contained in the class $\mathcal{L}^{(\alpha)}$ for which $\bar{F}(x)$ is regularly varying of index $-\alpha$ as $x \rightarrow \infty$. So, we have

$$
\frac{\bar{\Pi}_{\mathscr{H}}(u-y)}{\bar{\Pi}_{\mathscr{H}}(u)}=\frac{\bar{F}\left(\mathrm{e}^{u-y}\right)}{\bar{F}\left(\mathrm{e}^{u}\right)} \rightarrow \mathrm{e}^{\alpha y} \quad \text { as } u \rightarrow \infty \text { for each } y>0 .
$$

By [2, Theorem 1.5.6(ii)] we have the following Potter bound: for any given $\varepsilon \in(0, \alpha)$, there exists a constant $A(\varepsilon)>1$ such that, for all $r \geq 1$ and $s \geq 1$,

$$
\frac{\bar{F}(s)}{\bar{F}(r)} \leq A(\varepsilon) \max \left(\left(\frac{s}{r}\right)^{-\alpha+\varepsilon},\left(\frac{s}{r}\right)^{-\alpha-\varepsilon}\right) .
$$

Choose $\varepsilon \in(0, \alpha)$, take $\lambda>1$, and set $r=\lambda s$ then $s=\lambda r$ in this bound, to obtain

$$
\frac{1}{A(\varepsilon)} \lambda^{-\alpha-\varepsilon} \leq \frac{\bar{F}(\lambda r)}{\bar{F}(r)} \leq A(\varepsilon) \lambda^{-\alpha+\varepsilon} \quad \text { for all } r \geq 1 \text { and all } \lambda>1 \text {. }
$$


Since $\bar{\Pi}_{\mathscr{H}} \in \mathcal{f}^{(\alpha)}$, we thus have

$$
\frac{\bar{\Pi}_{\mathscr{H}}\left(\theta^{-1} \log x+u-y\right)}{\bar{\Pi}_{\mathscr{H}}(u-y)} \leq A(\varepsilon) x^{-(\alpha-\varepsilon) / \theta} \quad \text { for all } x>1 \text { and } u>y \geq 0 .
$$

Thus, furthermore, for all $x \geq 1$ and $0 \leq y<u$,

$$
\frac{\bar{\Pi}_{\mathscr{H}}\left(\theta^{-1} \log x+u-y\right)}{\bar{\Pi}_{\mathscr{H}}(u)} \leq A^{2}(\varepsilon) \mathrm{e}^{(\alpha+\varepsilon) y} x^{-(\alpha-\varepsilon) / \theta} .
$$

Using (4.6) and dominated convergence, we obtain, from (4.3),

$$
\lim _{u \rightarrow \infty} \frac{I(u, y)}{\bar{\Pi}_{\mathscr{H}}(u)}=\mathrm{e}^{\alpha y} \int_{1}^{\infty} x^{-\alpha / \theta} \mathrm{d} x=I(y) \text { for each } y>0 .
$$

Then, for each $a>0$ and $u>a$, write

$$
\int_{(0, u]}\left(\frac{I(u, y)}{\bar{\Pi}_{\mathscr{H}}(u)}-I(y)\right) V(\mathrm{~d} y)=\left[\int_{(0, a]}+\int_{(a, u]}\right]\left(\frac{I(u, y)}{\bar{\Pi}_{\mathscr{H}}(u)}-I(y)\right) V(\mathrm{~d} y) .
$$

Again, using (4.6) in (4.3) gives

$$
\begin{aligned}
\frac{I(u, y)}{\bar{\Pi}_{\mathscr{H}}(u)} & \leq A^{2}(\varepsilon) \mathrm{e}^{(\alpha+\varepsilon) y} \int_{1}^{\infty} x^{-(\alpha-\varepsilon) / \theta} \mathrm{d} x \\
& \leq \frac{\theta A^{2}(\varepsilon) \mathrm{e}^{(\alpha+\varepsilon) y}}{\alpha-\theta} \text { for all } u>y \geq 0 .
\end{aligned}
$$

By dominated convergence, the first integral on the right-hand side of (4.8) tends to 0 as $u \rightarrow \infty$, for each $a>0$. Since $I(y)=\theta \mathrm{e}^{\alpha y} /(\alpha-\theta)$ and $\delta_{\alpha}(V)=\int_{0}^{\infty} \mathrm{e}^{\alpha y} V(\mathrm{~d} y)$ is finite, the term $\int_{(a, u]} I(y) V(\mathrm{~d} y)$ in (4.8) tends to 0 as $u \rightarrow \infty$ and then $a \rightarrow \infty$.

This leaves us to deal in (4.8) with

$$
\int_{(a, u]} I(u, y) V(\mathrm{~d} y)=\int_{a}^{u} \bar{V}(y) g(u, y) \mathrm{d} y+\bar{V}(a) I(u, a)-\bar{V}(u) I(u, u),
$$

where we set

$$
\begin{aligned}
g(u, y) & =\frac{\partial}{\partial y} I(u, y) \\
& =\frac{\partial}{\partial y} \int_{u-y}^{\infty} \theta \mathrm{e}^{(z-u+y)} \bar{\Pi}_{\mathscr{H}}(z) \mathrm{d} z \\
& =\theta \bar{\Pi}_{\mathscr{H}}(u-y)+\theta^{2} \int_{u-y}^{\infty} \mathrm{e}^{\theta(z-u+y)} \bar{\Pi}_{\mathcal{H}}(z) \mathrm{d} z \\
& =\int_{(u-y, \infty)} \theta \mathrm{e}^{\theta(z-u+y)} \Pi_{\mathscr{H}}(\mathrm{d} z) .
\end{aligned}
$$

(Differentiating under the integral is valid here since $\int_{1}^{\infty} \mathrm{e}^{\alpha z} \bar{\Pi}_{\mathcal{H}}(z) \mathrm{d} z<\infty$ and $\theta<\alpha$.) We have to divide through by $\bar{\Pi}_{\mathscr{H}}(u), u>a$, in (4.9), and let $u \rightarrow \infty$ and then $a \rightarrow \infty$. We have, by (4.7),

$$
\lim _{u \rightarrow \infty} \frac{\bar{V}(a) I(u, a)}{\bar{\Pi}_{\mathscr{H}}(u)} \leq \frac{\theta \mathrm{e}^{\alpha a} \bar{V}(a)}{\alpha-\theta},
$$


and $\mathrm{e}^{\alpha a} \bar{V}(a) \rightarrow 0$ as $a \rightarrow \infty$, since $\delta_{\alpha}(V)<\infty$, so the term in $\bar{V}(a) I(u, a)$ in (4.9) presents no problem. Write the remainder of (4.9) as

$$
\begin{aligned}
\int_{a}^{u} \bar{V}(y) g(u, y) \mathrm{d} y-\bar{V}(u) I(u, u) \\
\quad=\int_{a}^{u}(\bar{V}(y)-\bar{V}(u)) g(u, y) \mathrm{d} y-\bar{V}(u) I(u, a) \\
\quad=\int_{0}^{u-a}(\bar{V}(u-y)-\bar{V}(u)) g(u, u-y) \mathrm{d} y+o\left(\bar{\Pi}_{\mathcal{H}}(u)\right) \quad \text { as } u \rightarrow \infty,
\end{aligned}
$$

where the ' $o$ ' term follows from (4.7) and $\bar{V}(u) \rightarrow 0$ as $u \rightarrow \infty$. Now, by (4.10),

$$
\begin{aligned}
g(u, u-y) & =\theta \bar{\Pi}_{\mathcal{H}}(y)+\theta^{2} \int_{y}^{\infty} \mathrm{e}^{\theta(z-y)} \bar{\Pi}_{\mathscr{H}}(z) \mathrm{d} z \\
& =\theta \int_{(y, \infty)} \mathrm{e}^{\theta(z-y)} \Pi_{\mathscr{H}}(\mathrm{d} z) \\
& =: h(y), \quad \text { say. }
\end{aligned}
$$

Decompose the integral in (4.11) into components over $(0, a]$ and $(a, u-a$ ], where $a<u / 2$, and divide by $\bar{V}(u)$. Since $\bar{V}(u) \sim \delta_{\alpha}^{2}(V) \bar{\Pi}_{\mathcal{H}}(u)$ as $u \rightarrow \infty$, by (2.5), the same bounds in (4.5) and (4.6) hold with $\bar{V}$ in place of $\bar{\Pi}_{\mathcal{H}}$, and $\bar{V}(u-y) / \bar{V}(u) \rightarrow \mathrm{e}^{\alpha y}$ as $u \rightarrow \infty$ for each $y \geq 0$. Then, by dominated convergence, for each $a>0$,

$$
\lim _{u \rightarrow \infty} \int_{0}^{a}\left(\frac{\bar{V}(u-y)}{\bar{V}(u)}-1\right) h(y) \mathrm{d} y=\int_{0}^{a}\left(\mathrm{e}^{\alpha y}-1\right) h(y) \mathrm{d} y,
$$

and this tends to $\int_{0}^{\infty}\left(\mathrm{e}^{\alpha y}-1\right) h(y) \mathrm{d} y$ as $a \rightarrow \infty$.

So it remains to deal with

$$
\begin{aligned}
0 & \leq \frac{1}{\bar{V}(u)} \int_{a}^{u-a}(\bar{V}(u-y)-\bar{V}(u)) h(y) \mathrm{d} y \\
& \leq \frac{1}{\bar{V}(u)} \int_{a}^{u-a} \bar{V}(u-y) h(y) \mathrm{d} y \\
& \leq \frac{c_{+}}{c_{-} \bar{\Pi}_{\mathscr{H}}(u)} \int_{a}^{u-a} \bar{\Pi}_{\mathscr{H}}(u-y) h(y) \mathrm{d} y,
\end{aligned}
$$

where we note from (4.12) that $h(y) \geq 0$. Here $c_{-} \bar{\Pi}_{\mathscr{H}}(z) \leq \bar{V}(z) \leq c_{+} \bar{\Pi}_{\mathscr{H}}(z)$ for some $0<c_{-} \leq c_{+}<\infty$ and all $z \geq a$, once $a$ is large enough, by (2.5). Now note that, as $y \rightarrow \infty$, by dominated convergence, and using the Potter bound,

$$
\begin{aligned}
\frac{h(y)}{\bar{\Pi}_{\mathscr{H}}(y)} & =\theta+\theta^{2} \int_{0}^{\infty} \mathrm{e}^{\theta z} \frac{\bar{\Pi}_{\mathscr{H}}(z+y)}{\bar{\Pi}_{\mathscr{H}}(y)} \mathrm{d} z \\
& \rightarrow \theta+\theta^{2} \int_{0}^{\infty} \mathrm{e}^{-(\alpha-\theta) z} \mathrm{~d} z \\
& =\frac{\alpha \theta}{\alpha-\theta} .
\end{aligned}
$$


Thus, for large $a$, we can upper bound the right-hand side of (4.13) by a multiple of

$$
\frac{1}{\bar{\Pi}_{\mathscr{H}}(u)} \int_{a}^{u-a} \bar{\Pi}_{\mathscr{H}}(u-y) \bar{\Pi}_{\mathcal{H}}(y) \mathrm{d} y,
$$

which we write as

$$
\frac{1}{\bar{\Pi}_{\mathcal{H}}(u)} \int_{a}^{u-a} \bar{\Pi}_{\mathcal{H}}(u-y)(-\mathrm{d} \bar{g}(y)) \mathrm{d} y,
$$

where $\bar{g}(y):=\int_{y}^{\infty} \bar{\Pi}_{\mathscr{H}}(z) \mathrm{d} z$ is, apart from a possible scale factor, the tail of a cumulative distribution function on $y \geq a$. Furthermore,

$$
\frac{\bar{g}(y)}{\bar{\Pi}_{\mathcal{H}}(y)}=\int_{0}^{\infty} \frac{\bar{\Pi}_{\mathscr{H}}(z+y)}{\bar{\Pi}_{\mathcal{H}}(y)} \mathrm{d} z \rightarrow \int_{0}^{\infty} \mathrm{e}^{-\alpha z} \mathrm{~d} z \quad \text { as } y \rightarrow \infty,
$$

by dominated convergence, and using (4.6) again. Integration by parts in (4.14) gives

$$
\frac{\bar{g}(a) \bar{\Pi}_{\mathscr{H}}(u-a)}{\bar{\Pi}_{\mathscr{H}}(u)}-\frac{\bar{g}(u-a) \bar{\Pi}_{\mathscr{H}}(a)}{\bar{\Pi}_{\mathscr{H}}(u-a)} \frac{\bar{\Pi}_{\mathscr{H}}(u-a)}{\bar{\Pi}_{\mathscr{H}}(u)}+\frac{1}{\bar{\Pi}_{\mathscr{H}}(u)} \int_{(a, u-a]} \bar{g}(u-y) \Pi_{\mathscr{H}}(\mathrm{d} y),
$$

in which the first two terms tend to 0 as $u \rightarrow \infty$ and then $a \rightarrow \infty$, because $\bar{g}(u)=O\left(\bar{\Pi}_{\mathscr{H}}(u)\right)$ as $u \rightarrow \infty$ and $\mathrm{e}^{\alpha a} \bar{\Pi}_{\mathcal{H}}(a) \rightarrow 0$ as $a \rightarrow \infty$. Replacing $\bar{g}(u-y)$ by a multiple of $\bar{\Pi}_{\mathcal{H}}(u-y)$ in the last term, as we may, finally leaves a multiple of

$$
\int_{(a, u-a]} \frac{\bar{\Pi}_{\mathscr{H}}(u-y)}{\bar{\Pi}_{\mathscr{H}}(u)} \Pi_{\mathscr{H}}(\mathrm{d} y) .
$$

This tends to 0 as $u \rightarrow \infty$ and then $a \rightarrow \infty$ by [9, Lemma 7.1]. (This is where special properties of the class $\delta^{(\alpha)}$ are used crucially.)

Putting the pieces together, we have arrived at

$$
\begin{aligned}
\lim _{u \rightarrow \infty} \mathrm{E} & {\left[\exp \left\{\theta\left(X_{\tau(u)}-u\right)\right\}-1 \mid \tau(u)<\infty\right] } \\
= & \lim _{u \rightarrow \infty} \frac{\bar{\Pi}_{\mathscr{H}}(u)}{\mathrm{P}(\tau(u)<\infty)} \int_{(0, u]} \frac{I(u, y)}{\bar{\Pi}_{\mathscr{H}}(u)} V(\mathrm{~d} y) \\
= & \frac{1}{q \delta_{\alpha}^{2}(V)}\left(\frac{\theta \delta_{\alpha}(V)}{\alpha-\theta}+\delta_{\alpha}^{2}(V) \int_{0}^{\infty}\left(\mathrm{e}^{\alpha y}-1\right) h(y) \mathrm{d} y\right) \\
= & \frac{\theta}{q(\alpha-\theta) \delta_{\alpha}(V)}+\frac{\theta}{q} \int_{0}^{\infty} \int_{0}^{y} \mathrm{e}^{\theta x}\left(\mathrm{e}^{\alpha(y-x)}-1\right) \mathrm{d} x \Pi_{\mathscr{H}}(\mathrm{d} y) .
\end{aligned}
$$

To complete the proof, we show that the right-hand side equals $\int_{(0, \infty)}\left(\mathrm{e}^{\theta x}-1\right) G(\mathrm{~d} x)$. Of course, this must follow by uniqueness of Laplace transforms. But the formulae are not obviously the same. To check, recall (2.8) and write

$$
\int_{1}^{\infty} \bar{G}\left(\theta^{-1} \log x\right) \mathrm{d} x:=I_{1}+I_{2}
$$

where

$$
I_{1}=\frac{q-\log \delta_{\alpha}(\mathscr{H})}{q} \int_{1}^{\infty} x^{-\alpha / \theta} \mathrm{d} x=\frac{\theta}{q(\alpha-\theta) \delta_{\alpha}(V)}
$$


(because $q-\log \delta_{\alpha}(\mathscr{H})=1 / \delta_{\alpha}(V)$; see [9, Proposition 5.1]) and

$$
\begin{aligned}
I_{2} & =\frac{1}{q} \int_{1}^{\infty} \mathrm{e}^{-\alpha \log x / \theta} \int_{(\log x / \theta, \infty)}\left(\mathrm{e}^{\alpha y}-\mathrm{e}^{\alpha \log x / \theta}\right) \Pi_{\mathscr{H}}(\mathrm{d} y) \mathrm{d} x \\
& =\frac{\theta}{q} \int_{0}^{\infty} \int_{0}^{y} \mathrm{e}^{\theta x}\left(\mathrm{e}^{\alpha(y-x)}-1\right) \mathrm{d} x \Pi_{\mathscr{H}}(\mathrm{d} y) .
\end{aligned}
$$

Thus, (2.10) is proved.

Proof of Theorem 3.1. Assume that $\lim _{t \rightarrow \infty} X_{t}=-\infty$ a.s.

(a) From (3.2) we obtain, for $u>0$ and $\beta>0$,

$$
\begin{aligned}
\int_{(u, \infty)} & v^{\beta} \mathrm{P}\left(u-X_{\tau(u)-} \in \mathrm{d} v, \tau(u)<\infty\right) \\
= & -\int_{(0, u]} \int_{v \in(u, \infty)} v^{\beta} \bar{\Pi}_{X}^{+}(v) \mathrm{d}_{v} \hat{V}(v-y) \mathrm{d}_{y} V(u-y) \\
= & \int_{(0, u]} \int_{v \in(u, \infty)} v^{\beta} \bar{\Pi}_{X}^{+}(v) \mathrm{d}_{v} \hat{V}(v-u+y) V(\mathrm{~d} y) \\
= & \int_{(0, u]} \int_{v \in(y, \infty)}(v+u-y)^{\beta} \bar{\Pi}_{X}^{+}(v+u-y) \hat{V}(\mathrm{~d} v) V(\mathrm{~d} y) \\
= & \int_{(0, u]}\left[\int_{v \in(y, u]}+\int_{v \in(u, \infty)}\right](v+u-y)^{\beta} \bar{\Pi}_{X}^{+}(v+u-y) \hat{V}(\mathrm{~d} v) V(\mathrm{~d} y) .
\end{aligned}
$$

The second component in (4.15) does not exceed

$$
2^{\beta} V(u) \int_{(u, \infty)} v^{\beta} \bar{\Pi}_{X}^{+}(v) \hat{V}(\mathrm{~d} v)
$$

and, on the other hand, is not smaller than

$$
V(u) \int_{(2 u, \infty)} v^{\beta} \bar{\Pi}_{X}^{+}\left(\frac{3 v}{2}\right) \hat{V}(\mathrm{~d} v) .
$$

Bertoin [1, p. 74] gave $\hat{V}(x) \asymp x / \hat{A}(x)$ for all $x \geq 0$, where $\hat{A}(x)=\int_{0}^{x} \bar{\Pi}_{\hat{H}}(y)$ d $y$ is finite and nonzero for all $x>0$. Assuming only that $\lim _{t \rightarrow \infty} X_{t}=-\infty$ a.s., Klüppelberg et al. [9], in the proof of their Proposition 5.4, showed that $\hat{A}(x) \asymp \int_{1}^{x} \bar{\Pi}_{X}^{-}(y) \mathrm{d} y \asymp A_{-}(x)$. Also, $A_{-}(\lambda x) \asymp A_{-}(x)$ for all $\lambda>0$. The renewal measure $\hat{V}(x)$ grows at most linearly with $x$, while $\lim _{x \rightarrow \infty} x^{\beta+1} \bar{\Pi}_{X}^{+}(x) / A_{-}(x)=0$ when the integral in (3.3) converges, as is easily checked since $x^{\beta+1} / A_{-}(x)$ is increasing in $x$. Thus, $I(v):=\int_{0}^{v} y^{\beta} \hat{V}(\mathrm{~d} y) \leq v^{\beta} \hat{V}(v) \asymp v^{\beta+1} / A_{-}(v)$. Integrating by parts gives

$$
\begin{aligned}
\int_{\eta}^{\infty} v^{\beta} \bar{\Pi}_{X}^{+}(\lambda v) \hat{V}(\mathrm{~d} v) & \leq \int_{\eta}^{\infty} I(v) \Pi_{X}(\lambda \mathrm{d} v) \\
& \leq \int_{\eta}^{\infty} v^{\beta} \hat{V}(v) \Pi_{X}(\lambda \mathrm{d} v) \\
& \asymp \int_{1}^{\infty} \frac{v^{\beta+1}}{A_{-}(v)} \Pi_{X}(\mathrm{~d} v) .
\end{aligned}
$$

Then we get finiteness of the $\beta$ th moment of the undershoot from (4.15). 
Conversely, assume that $\mathrm{E}\left[\left|X_{1}\right|\right]<\infty$ in addition to the finiteness of the expectation in (3.3). Then, since $X$ drifts to $-\infty$, we have $\mathrm{E}\left[\hat{H}_{1}\right]<\infty$, and, by the renewal theorem, $\hat{V}(x) / x \rightarrow c \in(0, \infty)$ for a constant $c>0$ as $x \rightarrow \infty$. Thus, we see that $I(x) \sim c^{\prime} x^{\beta+1}$ as $x \rightarrow \infty$ for a constant $c^{\prime} \in(0, \infty)$, and then the convergence of $\int_{(2 u, \infty)} v^{\beta} \bar{\Pi}_{X}^{+}(3 v / 2) \hat{V}(\mathrm{~d} v)$ implies the convergence of the integral in (3.3) (in which we can now delete the denominator function $A_{-}(x)$, since $A_{-}(\infty)<\infty$ now $)$.

(b) The proof of part (b) is similar to that of part (a).

Proof of Theorem 3.2. Assume that $\bar{\Pi}_{\mathscr{H}} \in \AA^{(\alpha)}$ for an $\alpha>0$.

(a) Some calculations from (3.2), and invoking Vigon's identity, (2.7), give, for $0<x<u$,

$$
\begin{aligned}
\mathrm{P}(u- & \left.X_{\tau(u)-}>x, \tau(u)<\infty\right) \\
= & \int_{(0, u)} \bar{\Pi}_{\mathscr{H}}(u-y) V(\mathrm{~d} y) \\
& \quad-\int_{y \in(u-x, u)} \int_{v \in[0, x-u+y]} \bar{\Pi}_{X}^{+}(v+u-y) \hat{V}(\mathrm{~d} v) V(\mathrm{~d} y) .
\end{aligned}
$$

Now

$$
\lim _{u \rightarrow \infty} \int_{(0, u)} \frac{\bar{\Pi}_{\mathscr{H}}(u-y)}{\bar{\Pi}_{\mathscr{H}}(u)} V(\mathrm{~d} y)=\delta_{\alpha}(V)+\delta_{\alpha}^{2}(V) \int_{(0, \infty)}\left(\mathrm{e}^{\alpha y}-1\right) \Pi_{\mathscr{H}}(\mathrm{d} y) .
$$

To prove this, consider, for $0<a<u$,

$$
\int_{(0, u)} \frac{\bar{\Pi}_{\mathscr{H}}(u-y)}{\bar{\Pi}_{\mathscr{H}}(u)} V(\mathrm{~d} y)=\left[\int_{(0, a]}+\int_{(a, u)}\right] \frac{\bar{\Pi}_{\mathscr{H}}(u-y)}{\bar{\Pi}_{\mathscr{H}}(u)} V(\mathrm{~d} y),
$$

in which the first integral tends to $\int_{(a, u]} \mathrm{e}^{\alpha y} V(\mathrm{~d} y)$ as $u \rightarrow \infty$ by dominated convergence, and this tends to $\int_{(0, \infty)} \mathrm{e}^{\alpha y} V(\mathrm{~d} y)=\delta_{\alpha}(V)$, finite, as $a \rightarrow \infty$. This leaves us to deal with

$$
\begin{aligned}
\int_{(a, u)} & \frac{\bar{\Pi}_{\mathscr{H}}(u-y)}{\bar{\Pi}_{\mathscr{H}}(u)}|\mathrm{d}(\bar{V}(y)-\bar{V}(u))| \\
= & \frac{\bar{\Pi}_{\mathscr{H}}(u-a)}{\bar{\Pi}_{\mathscr{H}}(u)}(\bar{V}(a)-\bar{V}(u))+\int_{(a, u)} \frac{(\bar{V}(y)-\bar{V}(u))}{\bar{\Pi}_{\mathscr{H}}(u)} \mathrm{d}_{y} \Pi_{\mathscr{H}}(u-y) .
\end{aligned}
$$

The first term tends to $\mathrm{e}^{\alpha a} \bar{V}(a)$ as $u \rightarrow \infty$, then to 0 as $a \rightarrow \infty$. By (2.5), the second term is asymptotic to

$$
\begin{aligned}
\delta_{\alpha}^{2}(V) & \int_{(0, u-a)}\left(\frac{\bar{V}(u-y)}{\bar{V}(u)}-1\right) \Pi_{\mathcal{H}}(\mathrm{d} y) \\
= & \delta_{\alpha}^{2}(V)\left[\int_{(0, a]}+\int_{(a, u-a]}\right]\left(\frac{\bar{V}(u-y)}{\bar{V}(u)}-1\right) \Pi_{\mathscr{H}}(\mathrm{d} y),
\end{aligned}
$$

in which the first component on the right-hand side tends to $\delta_{\alpha}^{2}(V) \int_{(0, a]}\left(\mathrm{e}^{\alpha y}-1\right) \Pi_{\mathcal{H}}(\mathrm{d} y)$ as $u \rightarrow \infty$, and this tends to $\delta_{\alpha}^{2}(V) \int_{(0, \infty)}\left(\mathrm{e}^{\alpha y}-1\right) \Pi_{\mathcal{H}}(\mathrm{d} y)$ as $a \rightarrow \infty$. The second component on the right-hand side is nonnegative and not greater than

$$
\frac{\delta_{\alpha}^{2}(V)}{\bar{V}(u)} \int_{(a, u-a]} \bar{V}(u-y) \Pi_{\mathscr{H}}(\mathrm{d} y) \leq c \int_{(a, u-a]} \frac{\bar{\Pi}_{\mathscr{H}}(u-y)}{\bar{\Pi}_{\mathscr{H}}(u)} \Pi_{\mathscr{H}}(\mathrm{d} y)
$$

for some $c>0$ and all large $a$. This tends to 0 as $u \rightarrow \infty$ and then $a \rightarrow \infty$ by [9, Lemma 7.1]. 
Going back to (4.17), we have to deal with

$$
\begin{aligned}
\int_{y \in(u-x, u)} & \int_{v \in[0, x-u+y]} \bar{\Pi}_{X}^{+}(v+u-y) \hat{V}(\mathrm{~d} v) V(\mathrm{~d} y) \\
= & -\int_{y \in(0, x)} \int_{v \in[0, x-y]} \bar{\Pi}_{X}^{+}(v+y) \hat{V}(\mathrm{~d} v) \mathrm{d}_{y} V(u-y) \\
= & -\int_{(0, x)} g_{x}(y) \mathrm{d}_{y} V(u-y),
\end{aligned}
$$

where

$$
g_{x}(y):=\int_{[0, x-y]} \bar{\Pi}_{X}^{+}(v+y) \hat{V}(\mathrm{~d} v) .
$$

Integrate by parts to obtain

$$
\begin{aligned}
\int_{(0, x)} g_{x}(y) \mathrm{d}_{y} V(u-y) & =-g_{x}(x) \bar{V}(u-x)+g_{x}(0) \bar{V}(u)+\int_{(0, x)} \bar{V}(u-y) g_{x}(\mathrm{~d} y) \\
& =\int_{(0, x)}(\bar{V}(u-y)-\bar{V}(u)) g_{x}(\mathrm{~d} y)-g_{x}(x)(\bar{V}(u-x)-\bar{V}(u)) \\
& \sim \bar{V}(u)\left(\int_{(0, x)}\left(\mathrm{e}^{\alpha y}-1\right) g_{x}(\mathrm{~d} y)-g_{x}(x)\left(\mathrm{e}^{\alpha x}-1\right)\right) \quad \text { as } u \rightarrow \infty \\
& =-\bar{V}(u) \int_{0}^{x} g_{x}(y) \mathrm{d}\left(\mathrm{e}^{\alpha y}\right),
\end{aligned}
$$

by dominated convergence, and using the uniform bounds in (4.6), applied to $\bar{V}$, as we may. Adjusting the constants gives (3.5).

(b) Take $\beta>0$. A calculation from (3.2) gives

$$
\int_{0}^{\infty} x^{\beta} \mathrm{P}\left(u-X_{\tau(u)-} \in \mathrm{d} x, \tau(u)<\infty\right)=\int_{(0, u]} g(u-y) V(\mathrm{~d} y),
$$

where, for $z>0$,

$$
g(z):=\int_{(0, \infty)}(x+z)^{\beta} \bar{\Pi}_{X}^{+}(x+z) \hat{V}(\mathrm{~d} x) .
$$

For $x>0, u>0$, and $0<\varepsilon<\alpha$,

$$
\frac{(x+u)^{\beta}}{u^{\beta}} \frac{\bar{\Pi}_{X}^{+}(x+u)}{\bar{\Pi}_{X}^{+}(u)} \leq 2^{\beta} A^{2}(\varepsilon)\left(\mathbf{1}_{\{x \leq u\}}+x^{\beta} \mathbf{1}_{\{x>u\}}\right) \mathrm{e}^{(-\alpha+\varepsilon) x},
$$

by the Potter bound, (4.6). Since $\hat{V}(x) \asymp x / \hat{A}(x)$ as $x \rightarrow \infty$, the integral $\int_{(0, \infty)} \mathrm{e}^{(-\alpha+\varepsilon) x} \hat{V}(\mathrm{~d} x)$ is finite. Note that, by (2.6) and (2.7),

$$
\hat{c}_{\alpha}:=\int_{(0, \infty)} \mathrm{e}^{-\alpha x} \hat{V}(\mathrm{~d} x)=\frac{1}{\hat{\kappa}(-\mathrm{i} \alpha)} .
$$

Thus, by dominated convergence, $g(u) \sim \hat{c}_{\alpha} u^{\beta} \bar{\Pi}_{X}^{+}(u) \sim u^{\beta} \bar{\Pi}_{\mathcal{H}}(u)$ as $u \rightarrow \infty$.

Now we want to normalise and take the limit as $u \rightarrow \infty$ in (4.19). Use the asymptotic for $g(u)$ to obtain

$$
\lim _{u \rightarrow \infty} u^{-\beta} \int_{(0, a]} \frac{g(u-y)}{\bar{\Pi}_{\mathscr{H}}(u)} V(\mathrm{~d} y)=\int_{(0, a]} \mathrm{e}^{\alpha y} V(\mathrm{~d} y) .
$$


This tends to $\delta_{\alpha}(V)$ as $a \rightarrow \infty$. Next, consider $\int_{(u-a, u]} g(u-y) V(\mathrm{~d} y)$, where $1<a<u$. For $0<z \leq a$,

$$
\begin{aligned}
g(z) & =\left[\int_{(0, a)}+\int_{(a, \infty)}\right](x+z)^{\beta} \bar{\Pi}_{X}^{+}(x+z) \hat{V}(\mathrm{~d} x) \\
& \leq(2 a)^{\beta} \int_{(0, \infty)} \bar{\Pi}_{X}^{+}(x+z) \hat{V}(\mathrm{~d} x)+2^{\beta} \int_{(1, \infty)} x^{\beta} \bar{\Pi}_{X}^{+}(x) \hat{V}(\mathrm{~d} x) \\
& \leq(2 a)^{\beta} \bar{\Pi}_{\mathcal{H}}(z)+O(1) .
\end{aligned}
$$

In the last inequality we used (2.7), (4.16), and the fact that $\int_{(1, \infty)} \mathrm{e}^{\alpha x} \Pi_{X}^{+}(\mathrm{d} x)$ is finite by (2.6). Hence,

$$
\begin{aligned}
& u^{-\beta} \int_{(u-a, u]} g(u-y) V(\mathrm{~d} y) \\
& \quad \leq u^{-\beta}\left((2 a)^{\beta} \int_{(u-a, u]} \bar{\Pi}_{\mathscr{H}}(u-y) V(\mathrm{~d} y)+O(\bar{V}(u-a)-\bar{V}(u))\right) \\
& \quad \leq u^{-\beta}\left((2 a)^{\beta} \int_{(u-a, u]} \bar{\Pi}_{\mathscr{H}}(u-y) V(\mathrm{~d} y)+\mathrm{e}^{\alpha a} O(\bar{V}(u))\right) .
\end{aligned}
$$

Note that the integral on the right-hand side is finite; in fact, it does not exceed 1, by (1.3). This integral equals

$$
\begin{aligned}
\int_{(u-a, u]} \bar{\Pi}_{\mathscr{H}}(u-y) \mathrm{d}(\bar{V}(y)-\bar{V}(u))= & \bar{\Pi}_{\mathscr{H}}(a)(\bar{V}(u-a)-\bar{V}(u)) \\
& +\int_{(0, a]}(\bar{V}(u-y)-\bar{V}(u)) \Pi_{\mathscr{H}}(\mathrm{d} y) .
\end{aligned}
$$

As $u \rightarrow \infty$, this is asymptotic to

$$
\bar{V}(u)\left(\bar{\Pi}_{\mathscr{H}}(a) \mathrm{e}^{\alpha a}+\int_{(0, a]}\left(\mathrm{e}^{\alpha y}-1\right) \Pi_{\mathcal{H}}(\mathrm{d} y)\right)=O(\bar{V}(u))=o\left(u^{\beta} \bar{V}(u)\right) .
$$

Thus, we obtain, for each $a>0$,

$$
\lim _{u \rightarrow \infty} u^{-\beta} \int_{(u-a, u]} \frac{g(u-y)}{\bar{\Pi}_{\mathscr{H}}(u)} V(\mathrm{~d} y)=0 .
$$

Finally, choose $a$ so large that $g(z) \leq 2 z^{\beta} \bar{\Pi}_{\mathscr{H}}(z)$ when $z \geq a$. Then

$$
\begin{aligned}
\frac{1}{u^{\beta} \bar{\Pi}_{\mathscr{H}}(u)} \int_{(a, u-a]} g(u-y) V(\mathrm{~d} y) & \leq \frac{2}{u^{\beta} \bar{\Pi}_{\mathscr{H}}(u)} \int_{(a, u-a]}(u-y)^{\beta} \bar{\Pi}_{\mathscr{H}}(u-y) V(\mathrm{~d} y) \\
& \leq \frac{2}{\bar{\Pi}_{\mathscr{H}}(u)} \int_{(a, u-a]} \bar{\Pi}_{\mathscr{H}}(u-y) V(\mathrm{~d} y) \\
& \sim \operatorname{constant} \int_{(a, u-a]} \frac{\bar{V}(u-y)}{\bar{V}(u)} V(\mathrm{~d} y) \quad \text { as } u \rightarrow \infty .
\end{aligned}
$$

The last expression tends to 0 as $u \rightarrow \infty$ and then $a \rightarrow \infty$ by [9, Lemma 7.1]. Collecting the terms together we obtain (3.6). Quite similar calculations give (3.7), proving part (c). 
Proof of Theorem 3.3. (a) For $0 \leq x \leq u, u>0$, we obtain, from (3.8),

$$
\begin{aligned}
\mathrm{P}(u & \left.-\bar{X}_{\tau(u)-} \leq x, \tau(u)<\infty\right) \\
& =\int_{(0, x]} \bar{\Pi}_{\mathscr{H}}(y) \mathrm{d}_{y} \bar{V}(u-y) \\
& =(\bar{V}(u-x)-\bar{V}(u)) \bar{\Pi}_{\mathcal{H}}(x)+\int_{(0, x]}(\bar{V}(u-y)-\bar{V}(u)) \Pi_{\mathcal{H}}(\mathrm{d} y) .
\end{aligned}
$$

Dividing by $\bar{V}(u)$ and letting $u \rightarrow \infty$ gives, on the right-hand side,

$$
\left(\mathrm{e}^{\alpha x}-1\right) \bar{\Pi}_{\mathscr{H}}(x)+\int_{(0, x]}\left(\mathrm{e}^{\alpha y}-1\right) \Pi_{\mathscr{H}}(\mathrm{d} y)=\alpha \int_{0}^{x} \bar{\Pi}_{\mathscr{H}}(y) \mathrm{e}^{\alpha y} \mathrm{~d} y .
$$

Then using (2.5) gives (3.9).

(b) From (3.8) we obtain, for $u>0$ and $\beta>0$,

$\mathrm{E}\left[\left(u-\bar{X}_{\left.\tau(u)_{-}\right)}\right)^{\beta}, \tau(u)<\infty\right]=\int_{0}^{u} y^{\beta} \bar{\Pi}_{\mathcal{H}}(y) \mathrm{d}_{y} \bar{V}(u-y)=\int_{0}^{u}(u-y)^{\beta} \bar{\Pi}_{\mathcal{H}}(u-y) V(\mathrm{~d} y)$.

Dividing by $u^{\beta} \bar{\Pi}_{\mathscr{H}}(u)$, this equals

$$
\int_{0}^{u}\left(1-\frac{y}{u}\right)^{\beta} \frac{\bar{\Pi}_{\mathscr{H}}(u-y)}{\bar{\Pi}_{\mathscr{H}}(u)} V(\mathrm{~d} y),
$$

and a proof just like that of (4.18) shows that this tends to $\delta_{\alpha}(V)$ as $u \rightarrow \infty$ (there is no extra term as in (4.18) because the factor of $(1-y / u)^{\beta}$ cancels the contribution near $\left.u\right)$. Thus, we obtain (3.10).

(c) From (3.8) we obtain, for $u>0$ and $\theta>0$,

$$
\frac{\mathrm{e}^{-\theta u} \mathrm{E}\left[\exp \left\{\theta\left(u-\bar{X}_{\tau(u)-}\right)\right\}, \tau(u)<\infty\right]}{\bar{\Pi}_{\mathscr{H}}(u)}=\int_{0}^{u} \mathrm{e}^{-\theta y} \frac{\bar{\Pi}_{\mathscr{H}}(u-y)}{\bar{\Pi}_{\mathscr{H}}(u)} V(\mathrm{~d} y) .
$$

The right-hand side converges to $\int_{(0, \infty)} \mathrm{e}^{(\alpha-\theta) y} V(\mathrm{~d} y)$ as $u \rightarrow \infty$, using dominated convergence, because, by the Potter bound in (4.5),

$$
\mathrm{e}^{-\theta y} \frac{\bar{\Pi}_{\mathscr{H}}(u-y)}{\bar{\Pi}_{\mathscr{H}}(u)} \mathbf{1}_{\{0 \leq y \leq u\}} \leq A(\varepsilon) \mathrm{e}^{-\theta y} \mathrm{e}^{(\alpha+\varepsilon) y}
$$

is integrable with respect to $V(\mathrm{~d} y)$ on $(0, \infty)$, once $\varepsilon<\theta$. Thus, we obtain (3.11).

\section{Acknowledgements}

We are grateful for the helpful comments of two anonymous referees which helped us improve the paper; in particular, Theorem 3.3 was added as a result of one query. The first author would especially like to thank Professor J. Gani for kind considerations during her visit at the Australian National University.

The work of the first author was supported by the Korea Research Foundation Grant KRF2006-214-C00012 funded by the Korean Government (MOEHRD) and by the SRC program of KOSEF (grant number R11-2000-073-00000). The work of the second author was partially supported by ARC Grant DP0664603. 


\section{References}

[1] Bertoin, J. (1996). Lévy Processes. Cambridge University Press.

[2] Bingham, N. H., Goldie, C. M. And Teugels, J. L. (1987). Regular Variation. Cambridge University Press.

[3] Doney, R. A. and Kyprianou, A. (2006). Overshoots and undershoots of Lévy processes. Ann. Appl. Prob. 16, 91-106.

[4] Doney, R. A. and Maller, R. A. (2004). Moments of passage times for Lévy processes. Ann. Inst. H. Poincaré Prob. Statist. 40, 279-297.

[5] Embrechts, P. And Goldie, C. M. (1982). On convolution tails. Stoch. Process. Appl. 13, 263-278.

[6] Embrechts, P., Goldie, C. M. and Veraverbeke, N. (1979). Subexponentiality and infinite divisibility. Z. Wahrscheinlichkeitsth. 49, 335-347.

[7] Grandell, J. (1991). Aspects of Risk Theory. Springer, New York.

[8] KlüPpelberg, C. (1989). Subexponential distributions and characterizations of related classes. Prob. Theory Relat. Fields 82, 259-269.

[9] Klüppelberg, C., Kyprianou, A. and Maller, R. (2004). Ruin probability and overshoots for general Lévy insurance risk processes. Ann. Appl. Prob. 14, 1766-1801.

[10] Kyprianou, A. (2005). Introductory Lectures on Fluctuations of Lévy Processes with Applications. Springer, Berlin.

[11] Sato, K. (1999). Lévy Processes and Infinitely Divisible Distributions. Cambridge University Press.

[12] Vigon, V. (2002). Votre Lévy rampe-t-il? J. London Math. Soc. 65, 243-256. 